\title{
COMMON STATIONARY POINTS FOR SET-VALUED MAPPINGS
}

\author{
M.S. KAHN \\ Department of Mathematics, Faculty of Science \\ Sultan Qaboos University, P.O. Box 32486 \\ Al-Khod, Muscat, Sultanate of Oman \\ K.R. RAO \\ Department of Mathematics, D.A.R. College \\ Nuzvid-521201, Krishna District, Andhra Pradesh, India \\ Y.J. CHO \\ Department of Mathematics, Gyeongsang National University \\ Jinju 660-701, Korea \\ (Received January 30, 1992 and in revised form May 25, 1992)
}

ABSTRACT. Several theorems on stationary points for set-valued mappings have obtained. These are improvements upon some earlier results due to Fisher.

KEY WORDS AND PHRASES. Generalized Hausdorff distance, nearly-densifying mappings, orbit, common stationary points.

1991 AMS SUBJECT CLASSIFICATION CODE. 54H25.

\section{INTRODUCTION AND PRELIMINARIES.}

In this paper, we prove several common stationary point theorems for four set-valued mappings, which are improvements upon some earlier results obtained by Fisher [1], [2], [3].

Let $(X, d)$ be a metric space and $C L(X)$ be the class of all nonempty closed subset of $X$. For $x \in X$ and $A \subseteq X$, let $D(x, A)=\inf \{d(x, y): y \in A\}$.

DEFINITION 1.1. For $A, B \in C L(X)$, define

$$
H(A, B)= \begin{cases}\max \left\{\sup _{x \in A} D(x, B), \sup _{y \in B} D(A, y)\right\}, & \text { if it exists, } \\ \infty, & \text { otherwise. }\end{cases}
$$

Then $H$ is called the generalized Hausdorff distance function for the class $C L(X)$ induced by the metric $d$.

DEFINITION 1.2. For $A, B \in C L(X)$, define $h: C L(X) \times C L(X) \rightarrow R^{+}$by

$$
h(A, B)= \begin{cases}\sup \{d(x, y): x \in A, y \in B\}, & \text { if it exists, } \\ \infty, & \text { otherwise }\end{cases}
$$

DEFINITION 1.3. A set-valued mapping $S: X \rightarrow C L(X)$ is said to be nearly-densifying if $\alpha(S(A))<\alpha(A)$ for any bounded and $S$-invariant subset of $X$ with $\alpha(A)>0$, where $\alpha$ is the Kuratowski's measure of non-compactness.

DEFINITION 1.4. Let $F, G, S, T: X \rightarrow C L(X)$ be set-valued mappings. For some $x \in X$, define the orbit $O(x)$ of $x$ by

$$
O(x)=\{y \in X: y=x \text { or } y=f(x) \text { for some } f \in \mathcal{T}\},
$$


$\tau$ being the subsemigroup generated by $F, G, S$ and $T$ in the semigroup of all self-mappings on $X$ with composition operation.

DEFINITION 1.5. A point $z$ is said to be a common stationary point of set-valued mappings $F$ and $G$ if $F z=\{z\}=G z$.

\section{THE MAIN RESULTS.}

Throughout this paper, for any set-valued mapping $S: X \rightarrow C L(X)$, we assume that all the powers of $S$ map $X$ into $C L(X)$. First of all, we prove the following crucial result to be used in the sequel.

LEMMA 2.1. Let $(X, d)$ be a compact metric space and $S: X \rightarrow C L(X)$ be a set-valued mapping such that $S^{\mathfrak{z}}$ is continuous with respect to the generalized Hausdorff distance function $H$ for some positive integer $i$. If $A=\cap_{k=1}^{\infty} S^{k_{1}}(X)$, then $S(A)=A$.

PROOF. Clearly, $S^{(k+1)}(X) \subset S^{k_{1}}(X)$ for $k=1,2, \cdots$. Also, $x \in X$ implies

$$
S x \subseteq A .
$$

Let $y \in A$. Then $y \in S^{(k+1)_{1}}(X)$ for $k=1,2, \cdots$, and so there exists $x_{k} \in S^{k_{1}}(X)$ such that $y=S^{1} x_{k}$ for $k=1,2, \cdots$. Since $X$ is compact, there exists a convergent subsequence $\left\{x_{k_{1}}\right\}$ of $\left\{x_{k}\right\}$ with the limit $z$. Further, since $\left\{x_{j}, x_{j+1}, \cdots\right\} \subseteq S^{J_{1}}(X)$ for $j=1,2, \cdots$, we have $z \in A$. Also, we have

$$
D\left(y, S^{2} z\right) \leq D\left(y, S^{i} x_{k_{\ell}}\right)+H\left(S^{4} x_{k} \ell^{i} z\right) .
$$

Letting $\quad \rightarrow 0$, we get $y \in S^{i}{ }$. Hence there exist $x_{1}, x_{i-1}, \cdots, x_{2} \in X$ such that $y \in S x_{1}$, $x_{1} \in S x_{1-1}, \cdots, x_{3} \in S x_{2}$, and $x_{2} \in S z$. By (1.1), since $z \in A$, it follows that $S z \subseteq A$ and so $x_{2} \in A$. A repeated application of (1.1) yields that $x_{i} \in A$. Therefore, we have $y \in S x$ for some $x \in A$. Thus, $A \subseteq S(A)$. From this and (1.1), we conclude that $S(A)=A$. This completes the proof.

Now, we are in a position to present our main results. We denote

$$
M\left(x, y, F^{p}, G^{q}, S^{s}, T^{t}\right)=\max \left\{h\left(S^{s} x, T^{t} y\right), h\left(S^{s} x, F^{p} x\right), h\left(T^{t} y, G^{q} y\right), h\left(S^{s} x, G^{q} y\right), h\left(T^{t} y, F^{p} x\right)\right\}
$$

and

$$
m\left(x, y, F^{p}, G^{q}, S^{s}, T^{t}\right)=\max \left\{h\left(S^{s} x, T^{t} y\right), h\left(S^{s} x, G^{q} y\right), h\left(T^{t} y, F^{p} x\right)\right\},
$$

where $p, q, s$ and $t$ are positive fixed integers.

THEOREM 2.1. Let $(X, d)$ be a complete metric space and $F, G, S, T: X \rightarrow C L(X)$ be set-valued mappings such that

(2.1) $F, G, S, T$ and $(F G)^{i}$ are continuous with respect to the distance function $H$ for some positive integer $i$. Also, $F, G, S$ and $T$ are nearly-densifying,

(2.2) for some $x_{o} \in X$, the orbit $O\left(x_{o}\right)$ is bounded,

(2.3) $H\left(F^{p} x, G^{q} y\right)<M\left(x, y, F^{p}, G^{q}, S^{\boldsymbol{s}}, T^{t}\right)$,

(2.4) $F G=G F,(F G)^{i} S^{s}=S^{s}(F G)^{i}$ and $(F G)^{i} T^{t}=T^{t}(F G)^{i}$.

Then $F, G, S$ and $T$ have a unique common stationary point $z$ in $X$.

PROOF. Putting $A=O\left(x_{0}\right)$, we have clearly $I(A)=A$ for $I \in\{F, G, S, T\}$. Also, the continuity of set-valued mappings $F, G, S$ and $T$ yields that $I(\bar{A}) \subseteq \bar{A}$ for $I \in\{F, G, S, T\}$. Further, we have $A=\left\{x_{o}\right\} \cup F(A) \cup G(A) \cup S(A) \cup T(A)$. Thus, $\alpha(A)=\max \left\{\alpha\left(x_{o}\right), \alpha(F(A)), \alpha(G(A)), \alpha(S(A)), \alpha(T(A))\right\}$ and also $\bar{A}$ is compact. Now, define $B=\cap_{n=1}^{\infty}(F G)^{i}(\bar{A})$. Then $B$ is compact. By Lemma 2.1, $(F G)(B)=B$ and the condition (2.4) ensures that $F(B)=B=G(B), S^{s}(B) \subseteq B$ and $T^{t}(B) \subseteq B$. Since $B$ is compact, there exist $x_{1}, x_{2} \in B$ such that $d\left(x_{1}, x_{2}\right)=\sup \{d(x, y): x, y \in B\}=\delta(B)$, say. Also, there exist $w_{1}, w_{2} \in B$ such that $x_{1} \in F^{p} w_{1}$ and $x_{2} \in G^{q} w_{2}$. Suppose that $\delta(B)>0$. Then, by (2.3), we 
have

$$
\begin{aligned}
\delta(B) & =d\left(x_{1}, x_{2}\right) \leq H\left(F^{p} w_{1}, G^{q} w_{2}\right) \\
& <M\left(w_{1}, w_{2}, F^{p}, G^{q}, S^{p}, T^{t}\right) \\
& \leq \delta(B),
\end{aligned}
$$

which is a contradiction. Thus, $\delta(B)=0$ and hence $B=\{z\}$, say. Therefore, $z$ is a common stationary point of $F, G, S$ and $T$. The uniqueness of $z$ follows from condition (2.3). This completes the proof.

THEOREM 2.2. Let $(X, d)$ be a compact metric space and $F, G, S, T: X \rightarrow C L(X)$ be set-valued mappings such that

(2.5) $(F G)^{4}$ is continuous for some positive integer $i$,

(2.6) $H\left(F^{p} x, G^{q} y\right)<M\left(x, y, F^{p}, G^{q}, S^{\bullet}, T^{t}\right)$ whenever the left-hand side is positive,

(2.7) $F G=G F,(F G)^{i} S^{\boldsymbol{D}}=S^{\boldsymbol{e}}(F G)^{i}$ and $(F G)^{i} T^{t}=T^{t}(F G)^{i}$.

Then $F, G, S$ and $T$ have a unique common stationary point $z$ in $X$. Further, $z$ is the unique common stationary point of $F$ and $G$.

PROOF. If we put $B=\cap_{n=1}^{\infty}(F G)^{i}(X)$, as in the proof of Theorem 2.1, we have $B=\{z\}$ and $z$ is a unique common stationary point of $F, G, S$ and $T$. Since any common stationary point of $F$ and $G$ is a point of $B=\{z\}$, it follows that $z$ is the unique common stationary point of $F$ and $G$. This completes the proof.

REMARK. Theorem 2 of Fisher [2] and theorems in Fisher [3] follow as corollaries of our Theorem 2.2. In fact, our theorem can be regarded as an improvement over the above theorems due to Fisher.

THEOREM 2.3. Let $(X, d)$ be a complete metric space and $F, G, S, T: X \rightarrow C L(X)$ be set-valued mappings such that

(2.8) $F, G, S, T, F^{i}$ and $G^{J}$ are continuous with respect to the distance function $H$ for some positive integers $i$ and $j$. Also, $F, G, S$ and $T$ are nearly-densifying,

(2.9) for some $x_{o} \in X$, the orbit $O\left(x_{0}\right)$ is bounded,

(2.10) $H\left(F^{p} x, G^{q} y\right)<m\left(x, y, F^{p}, G^{q}, S^{s}, T^{t}\right)$ whenever the left-hand side is positive,

(2.11) $S^{s} F^{t}=F^{i} S^{t}$ and $T^{t} G^{q}=G^{q} T^{t}$.

Then $F, G, S$ and $T$ have a unique common stationary point $z$ in $X$.

PROOF. Let $A=O\left(x_{o}\right)$. Then as in the proof of Theorem 2.1, $\bar{A}$ is compact. If we define

$$
B=\cap_{n=1}^{\infty} F^{i}(A) \text { and } K=\cap_{n=1}^{\infty} G^{j}(A) \text {, }
$$

by Lemma 2.1, $F(B)=B$ and $G(K)=K$. Also, it follows that $B$ and $K$ are compact subsets of $X$. By the condition (2.11), also we have $S^{\ell}(B) \subseteq B$ and $T^{t}(K) \subseteq K$. Then, there exist $x_{1}, w_{1} \in B$ and $y_{1}, y_{2} \in K$ such that

$$
d\left(x_{1}, y_{1}\right)=\sup \{d(x, y): x \in B, y \in K\}=\delta(B, K), \text { say, }
$$

with $x_{1} \in F^{p} w_{1}$ and $y \in G^{q} w_{2}$. Suppose that $\delta(B, K)>0$. Then, by the condition (2.10), we have

$$
\begin{aligned}
\delta(B, K) & =d\left(x_{1}, y_{1}\right) \\
& \leq H\left(F^{p} w_{1}, G^{q} w_{2}\right) \\
& <m\left(w_{1}, w_{2}, F^{p}, G^{q}, S^{\boldsymbol{t}}, T^{t}\right) \\
& \leq \delta(B, K),
\end{aligned}
$$


which is a contradiction. Therefore, $\delta(B, K)=0$ and $B=K=\{z\}$. Thus $z$ is a common stationary point of $F, G, S$ and $T$. The uniqueness of $z$ follows easily from the condition (2.10). This completes the proof.

THEOREM 2.4. Let $(X, d)$ be a compact metric space and $F, G, S, T: X \rightarrow C L(X)$ be set-valued mappings such that

(2.12) $F^{\prime}$ and $G^{J}$ are continuous with respect to the distance function $H$ for some positive integers $i$ and $j$,

(2.13) $H\left(F^{p} x, G^{q} y\right)<m\left(x, y, F^{p}, G^{q}, S^{s}, T^{\ell}\right)$ whenever the left-hand side is positive,

(2.14) $F^{t} S^{s}=S^{s} F^{t}$ and $G^{q} T^{t}=T^{t} G^{q}$.

Then $F, G, S$ and $T$ have a unique common stationary point $z$ in $X$. Further, $z$ is the unique common stationary point of the pairs $F, S$ and $G, T$. Also, $z$ is the unique common stationary point of $F$ and $G$.

PROOF. Let $B=\cap_{n=1}^{\infty} F^{2} n(X)$ and $K=\cap_{n=1}^{\infty} G^{j_{n}}(X)$. Then as in the proof of Theorem 2.3, we get $B=K=\{z\}$ and $z$ is a unique common stationary point of $F, G, S$ and $T$. Since any stationary point of $F$ is a point of $B=\{z\}$ and any stationary point of $G$ is a point of $K=\{z\}$, it follows that $z$ is the unique stationary point of $F$ as well as of $G$. This completes the proof.

REMARK. Theorem 5 of Fisher [1] follows as a corollary of our Theorem 2.3.

ACKNOWLEDGEMENT. We are grateful to Professor B. Fisher for providing us reprints of his papers which motivated the present work. Thanks are also due to Professor S. Sessa and the referee for their useful comments on the earlier version of our paper.

\section{REFERENCES}

1. FISHER, B., Four mappings with a common fixed point, J. Univ. Kuwait (Sci.), 8 (1981), 131-138.

2. FISHER, B., Three mappings with a common fixed point, Math. Sem. Notes, Kobe Univ., 10 (1982), 298-302.

3. FISHER, B., A common fixed point theorem for four mappings on a compact metric space, Inst. Math. Acad. Sinica, 12 (1984), 249-252. 


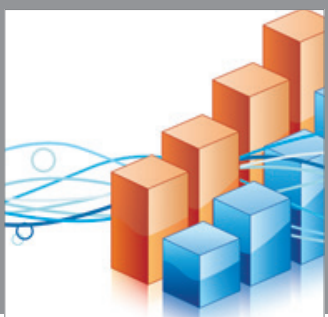

Advances in

Operations Research

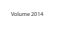

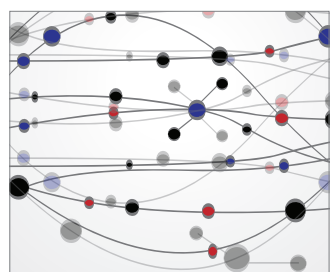

\section{The Scientific} World Journal
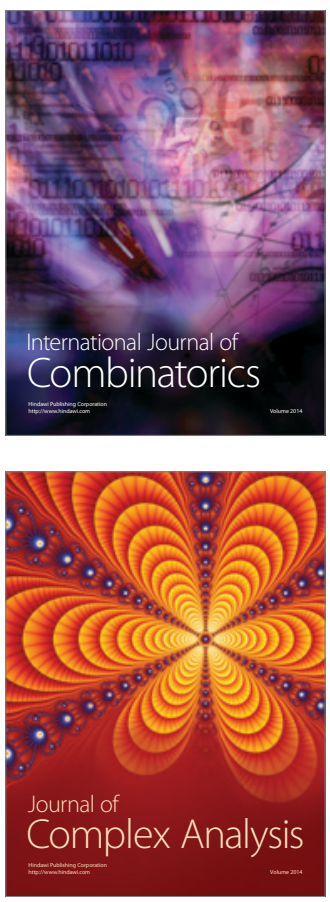

International Journal of

Mathematics and

Mathematical

Sciences
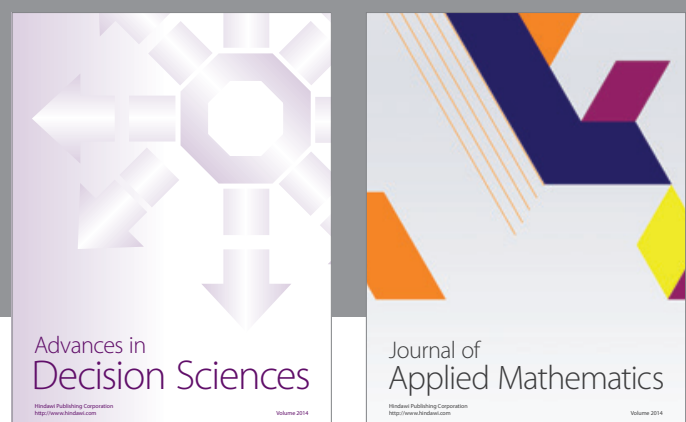

Journal of

Applied Mathematics
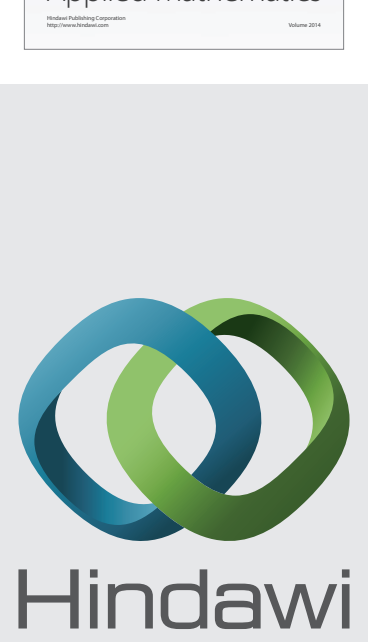

Submit your manuscripts at http://www.hindawi.com
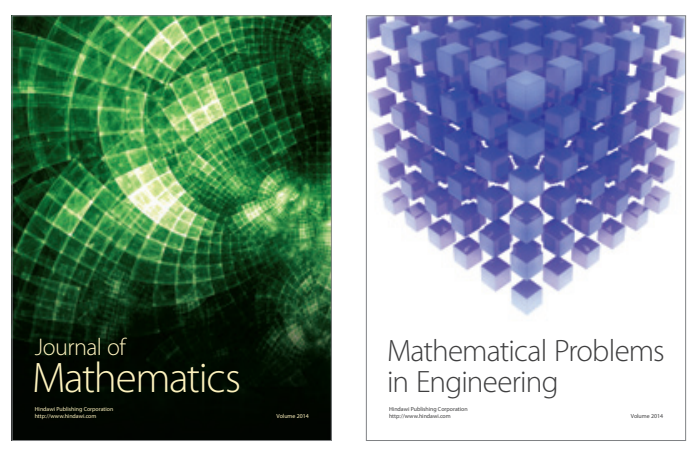

Mathematical Problems in Engineering
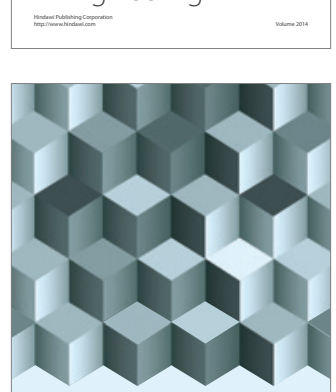

Journal of

Function Spaces
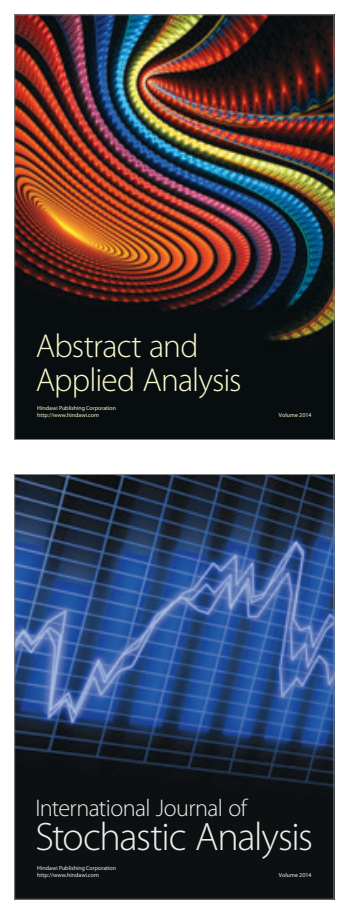

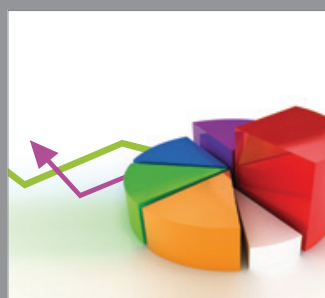

ournal of

Probability and Statistics

Promensencen
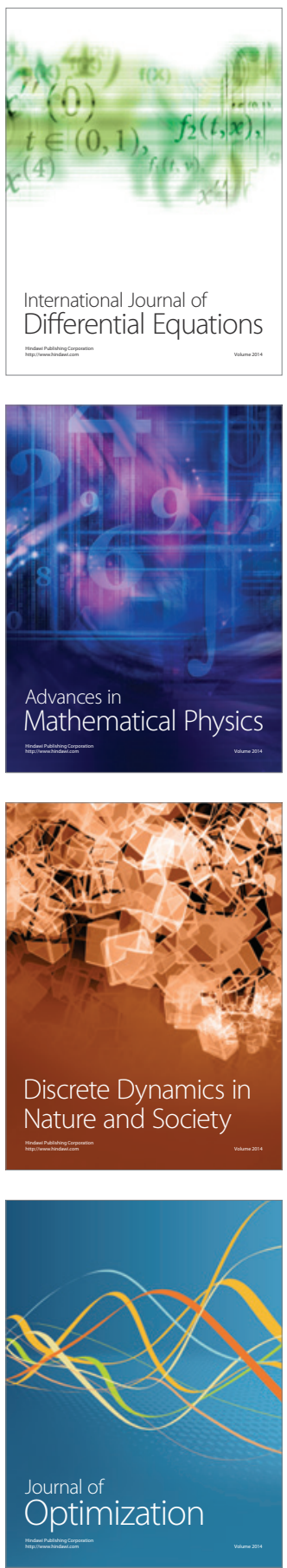